\title{
A Case of Refractory Polymyositis Successfully Treated With Abatacept Monotherapy
}

\author{
Mehmet ÇAĞLAYAN(D, Hüseyin IŞIK(D), Pelin OKTAYOGLU(D) \\ Department of Physical Medicine and Rehabilitation, Dicle University Faculty of Medicine, Diyarbakır, Turkey
}

\begin{abstract}
Polymyositis (PM) is an autoimmune disease progressing in the form of a break down of the muscles that is induced by chronic inflammation in skeletal muscles. Muscle weakness is painless and concentrates on proximal muscles, involving the pectoral and pelvic girdle. If the disease is not treated properly, it may progress and lead to a considerable decrease in the quality of life. Its conventional treatment involves drugs that suppress inflammation such as steroids, methotrexate, azathioprine, and intravenous immunoglobulin. However, conventional treatment may prove insufficient to halt the progression of the disease and offer only a limited improvement because of the adverse effects it causes in some patients. In this article, we present a 48-year-old female patient diagnosed with PM nearly 13 years ago that did not sufficiently respond to the pharmaceutical agents that were indicated for the conventional treatment of the disease and developed femoral head avascular necrosis because of the treatment and was, in the end, successfully treated with abatacept monotherapy at our clinic.

Keywords: Abatacept, monotherapy, polymyositis.
\end{abstract}

Idiopathic inflammatory myopathies (IIMs) are diseases of connective tissues with unknown etiologies that progress with mononuclear cell infiltration in muscles. Among all IIMs, polymyositis $(\mathrm{PM})$ is an autoimmune disease of connective tissues that manifests itself with muscle weakness. PM presents with bilateral symmetric proximal muscle weakness that progresses over weeks to months. Neck flexor weakness coupled with pharyngeal and laryngeal muscle involvement can lead to dysphonia. ${ }^{1}$

The incidence of $\mathrm{PM}$, which is twice more common among females compared to males, is 1 in $100,000 .^{2}$ Its etiology involves both genetic and environmental factors. Its progression may vary from mere involvement of the musculoskeletal system to its combination with the involvement of the heart, skin or lungs. Its conventional treatment requires the administration of intravenous immunoglobulin (IVIG) as well as inflammatory-suppressive drugs, including steroids, methotrexate and azathioprine. ${ }^{2,3}$ Glucocorticoids are regarded as the cornerstones of the treatment; however, some patients may require adjunctive immunosuppressive agents because of insufficient response to glucocorticoids, exacerbations upon glucocorticoid tapering or glucocorticoid-related adverse events.

On the other hand, such modalities of treatment may prove insufficient to halt the progression of the disease and offer only a limited improvement because of the adverse effects they cause in some patients. A large number of biological agents have been put to trial in an effort to treat this disease, including tumor necrosis factor-alpha (TNF- $\alpha$ ) blockers; however, the trials did not

Received: September 13, 2019 Accepted: September 20, 2019 Published online: February 07, 2020

Correspondence: Mehmet Çağlayan, MD. Dicle Üniversitesi Tıp Fakültesi Fiziksel Tıp ve Rehabilitasyon Anabilim Dalı, 21280 Sur, Diyarbakır, Türkiye. Tel: +90 412 - 2488001 / 4572 e-mail: ftrmehmet63@hotmail.com 
give promising results to be referenced in the relevant guidelines. Abatacept is a recombinant CTLA4-immunoglobulin (Ig) fusion protein and an agent that selectively suppresses T-cell activation through the cluster of differentiation (CD) $28: C D 80 / 86$ pathway. Even though the literature contains numerous studies (case studies and series of a small number of cases) concerning the use of abatacept in the treatment of PM, none of such studies employed this agent in the form of a monotherapy. ${ }^{3-6}$

In this article, we present a case diagnosed with PM that did not sufficiently respond to neither biological agents, namely rituximab, nor pharmaceutical agents that were indicated for the conventional treatment of the disease and developed femoral head avascular necrosis because of the corticosteroid treatment.

\section{CASE REPORT}

A 48-year-old female patient presented to our outpatient clinic two years ago with complaints of severe muscle weakness. She stated that muscle weakness had been troubling her for more than 10 years and she had presented to a hospital with muscle weakness, fatigue, and respiratory distress at the time. The epicrisis drafted for the patient back then showed the proximal muscle strength in both upper and lower extremities to be between $2 / 5$ and $3 / 5$ as identified in physical examination. No family history was indicated in the given report. According to the laboratory analyses, the patient had a creatine kinase (CK) level of 4,267 (29-200 U/L) and tested negative for antinuclear antibodies and extractable nuclear antigens. Electromyography findings were suggestive of inflammatory myopathy in the proximal muscles of both lower and upper extremities. Furthermore, muscle biopsy performed back then led to the observation of necrosis in the deltoid muscle fibers, which was considered to be suggestive of PM. Neither thoracic computed tomography nor electrocardiographic examination indicated pathologies. At the time, the patient was put on a $1,000 \mathrm{mg} /$ day of methylprednisolone treatment for three days, and the treatment was continued with $1 \mathrm{mg} / \mathrm{kg} /$ day of methylprednisolone and $15 \mathrm{mg} /$ week of methotrexate, and the dose of methylprednisolone was gradually reduced to $4 \mathrm{mg} /$ day. The patient benefited from the treatment to a certain extent; however, she had to come back to the clinic within eight months because of recurrence of the previous complaints, upon which hydroxychloroquine was added to her treatment regimen. Nine years ago, the clinic shifted to $1 \mathrm{mg} / \mathrm{kg} /$ day of prednisolone and $3 \mathrm{mg} / \mathrm{kg} /$ day of azathioprine because of insufficient improvement in the patient's condition. Seven years ago, subcutaneous administration of $15 \mathrm{mg} /$ week of methotrexate was added to her treatment regimen, upon which the patient suffered further deterioration and muscle weakness and received a course of intravenous immunoglobulin, followed by $40 \mathrm{mg} /$ day of leflunomide in the next years. She also received $1,000 \mathrm{mg}$ of intravenous ritixumab twice with a two-week interval nearly a year ago. No positive effects were observed after these treatments, and the $\mathrm{CK}$ levels of the patient was identified to be above $1,000 \mathrm{U} / \mathrm{L}$ all the time, ranging between 1,000 and $4,000 \mathrm{U} / \mathrm{L}$. A written informed consent was obtained from the patient.

The patient received a total hip replacement because of aseptic necrosis in the right femoral head that developed due to the use of steroids. Our physical examination indicated the muscle strength to be $1 / 5$ in the proximal side of the bilateral upper extremities and $2 / 5$ in the proximal side of the lower extremities. There were no marked findings of atrophy in the muscles. We found the body mass index to be $30 \mathrm{~kg} / \mathrm{m}^{2}$. With a level of $\mathrm{CK}$ at 3,025 U/L, the patient was put on a monotherapy regimen of $125 \mathrm{mg} /$ week of abatacept. After two months of treatment, muscle strength was found to be $3 / 5$ in the upper extremities and $4 / 5$ in the lower extremities, and the CK level dropped to $1,950 \mathrm{U} / \mathrm{L}$ (Figure 1). Muscle strength was found to be $3 / 5$ in the upper extremities and $4 / 5$ in the lower extremities in the fourth month of the treatment, with $\mathrm{CK}$ level coming down to $1,200 \mathrm{U} / \mathrm{L}$. Her examinations at month eight showed a muscle strength of $4 / 5$ in both upper and lower extremities and a $\mathrm{CK}$ level of $1,003 \mathrm{U} / \mathrm{L}$. The patient was put on resistance exercises. The whole cycle of a 12 -month treatment was completed in November 2018 with full muscle strength achieved and a CK level of $484 \mathrm{U} / \mathrm{L}$. The patient, who is 


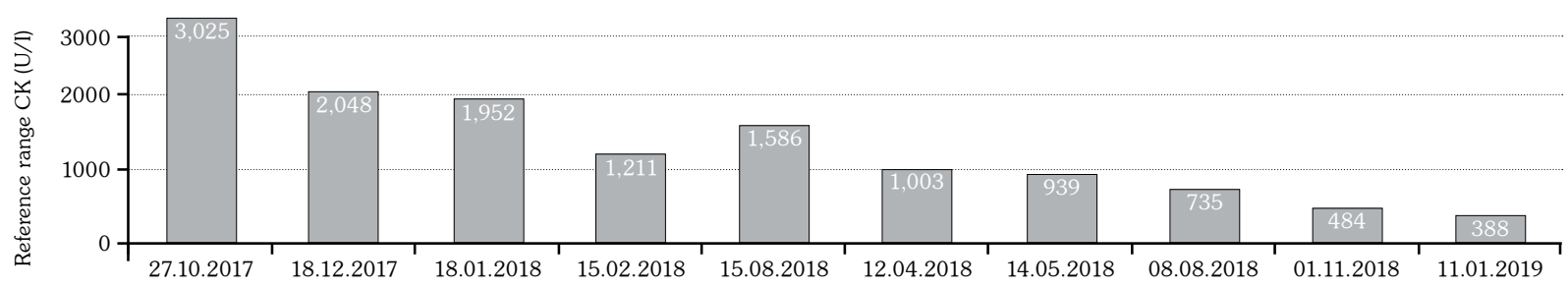

Figure 1. Creatine kinase levels at the patient's control dates.

CK: Creatine kinase.

being regularly followed-up, is still receiving a monotherapy of $125 \mathrm{mg} /$ week of abatacept, and her condition is stable.

\section{DISCUSSION}

Polymyositis is an autoimmune disease progressing in the form of a breakdown of the muscles that is induced by chronic inflammation in skeletal muscles. Although its etiology is unknown, the most commonly acknowledged hypothesis is the destruction of muscle fibers mediated by T lymphocytes. ${ }^{7}$ Biopsy findings are characterized by a dense infiltration of muscle fibers by inflammatory cells composed mostly of CD8+ $\mathrm{T}$ cells and macrophages and the damage arising from such infiltration. ${ }^{8}$

Steroids constitute the building block of the treatment. On the other hand, addition of conventional immunosuppressive agents such as methotrexate or azathioprine to the treatment regimen leads to improved efficacy and reduction in the dosage of steroids required. Patients refractory to treatment are, on the other hand, treated with IVIG. ${ }^{9,10}$ Nevertheless, such drugs may either cause adverse effects or prove insufficient to halt the progression of the disease. Conventional immunosuppressive agents can lead to such complications as osteoporosis, bone marrow suppression, hyperglycemia and aseptic necrosis. Our case had, in fact, benefitted from steroids; however, she had also developed femoral head aseptic necrosis because of these agents. Despite administration of conventionally indicated drugs, high CK levels and muscle weakness persisted in the patient. Use of biological agents is recommended in cases refractory to the conventional treatment of PM. Rituximab is the most commonly trialed biological agent in this context. A previous comprehensive study (rituximab in myositis) showed rituximab to be effective in the majority of refractory patients. ${ }^{11}$ Rituximab was reported to be a good option in patients who were not respondent to or fit for conventional treatment.

$\mathrm{T}$ lymphocytes are observed to play a key role in both the onset and the persistence of inflammation in PM. This observation leads to the consideration that abatacept may be effective in the treatment of this disease as it is a recombinant CTLA4-Ig fusion protein that provides for the inhibition of $\mathrm{T}$ lymphocyte costimulation. ${ }^{12,13}$ The literature contains numerous studies (case studies and series of a small number of cases) concerning the use of abatacept in the treatment of PM. However, such studies all combined abatacept with other immunosuppressive agents. Tjärnlund et al. ${ }^{4}$ administered a combination of abatacept and conventional disease-modifying antirheumatic drug in 20 patients diagnosed with PM and dermatomyositis and reported improvements in the results of manual muscle testing and patient activity in half of the patients. Furthermore, Musuruana and Cavallasca ${ }^{5}$ reported a dramatic response from a patient receiving methotrexate and azathioprine combined with abatacept. In a case report, Maeshima et al. $^{6}$ found abatacept to be effective in a patient that did not respond to any biological agents, including a TNF blocker and tocilizumab. Nevertheless, none of these studies administered abatacept in the form of a monotherapy.

The present case had a CK level decreasing from 3,025 to 2,048 within two months after the initiation of abatacept monotherapy. After about two years, the CK level dropped to $484 \mathrm{U} / \mathrm{L}$, and 
muscle strength was restored to normal levels. A good response to treatment was secured in two to four months in studies combining abatacept with another drug. Despite the late response observed with the present case, the monotherapy helped us avoid potential adverse effects that could have been caused by a combination of drugs.

Most patients with PM respond to methotrexate; however, this agent should be avoided in patients that have interstitial lung disease and test positive for anti-Jo-1 antibodies due to its possible result of pulmonary fibrosis. ${ }^{14,15}$ Previous studies report beneficial effects of azathioprine, ${ }^{16}$ IVIG,${ }^{17}$ mycophenolate mofetil, ${ }^{18}$ TNF- $\alpha$ blockers ${ }^{19}$ and plasmapheresis. ${ }^{20}$

The preferred management of PM has traditionally been with corticosteroids; however, some patients require adjunctive immunosuppressive agents because of insufficient response to glucocorticoids. The administration of abatacept monotherapy in patients with PM may be considered as a treatment option because it allows for monotherapy in patients with PM that cannot be managed with biological or other immunsupressive agents and develop adverse effects because of corticosteroids.

In conclusion, abatacept monotherapy can safely be used in patients with PM that do not respond to conventional treatment or develop treatment-related complications, with due consideration given to the pathogenesis of the disease and the action mechanism of the agent. There is a need for further comprehensive studies with large sample sizes to better evaluate the efficacy of abatacept monotherapy in PM.

\section{Declaration of conflicting interests}

The authors declared no conflicts of interest with respect to the authorship and/or publication of this article.

\section{Funding}

The authors received no financial support for the research and/or authorship of this article.

\section{REFERENCES}

1. Ernste FC, Reed AM. Idiopathic inflammatory myopathies: current trends in pathogenesis, clinical features, and up-to-date treatment recommendations. Mayo Clin Proc 2013;88:83-105.
2. Dalakas MC, Hohlfeld R. Polymyositis and dermatomyositis. Lancet 2003;362:971-82.

3. Kerola AM, Kauppi MJ. Abatacept as a successful therapy for myositis-a case-based review. Clin Rheumatol 2015;34:609-12.

4. Tjärnlund A, Tang Q, Wick C, Dastmalchi M, Mann $\mathrm{H}$, Tomasová Studynková J, et al. Abatacept in the treatment of adult dermatomyositis and polymyositis: a randomised, phase IIb treatment delayed-start trial. Ann Rheum Dis 2018;77:55-62.

5. Musuruana JL, Cavallasca JA. Abatacept for treatment of refractory polymyositis. Joint Bone Spine 2011;78:431-2.

6. Maeshima K, Kiyonaga Y, Imada C, Iwakura M, Hamasaki H, Haranaka M, et al. Successful treatment of refractory anti-signal recognition particle myopathy using abatacept. Rheumatology (Oxford) 2014;53:379-80.

7. Grundtman C, Lundberg IE. Pathogenesis of idiopathic inflammatory myopathies. Curr Rheumatol Rep 2006;8:188-95.

8. Mammen AL. Dermatomyositis and polymyositis: Clinical presentation, autoantibodies, and pathogenesis. Ann N Y Acad Sci 2010;1184:134-53.

9. Aggarwal R, Oddis CV. Therapeutic advances in myositis. Curr Opin Rheumatol 2012;24:635-41.

10. Carstens PO, Schmidt J. Diagnosis, pathogenesis and treatment of myositis: recent advances. Clin Exp Immunol 2014;175:349-58.

11. Oddis CV, Reed AM, Aggarwal R, Rider LG, Ascherman $\mathrm{DP}$, Levesque MC, et al. Rituximab in the treatment of refractory adult and juvenile dermatomyositis and adult polymyositis: a randomized, placebo-phase trial. Arthritis Rheum 2013;65:314-24.

12. Iannone $F$, Lapadula $G$. The inhibitor of costimulation of T cells: abatacept. J Rheumatol Suppl 2012;Suppl 89:100-2.

13. Lundberg IE, Vencovsky J, Alexanderson H. Therapy of myositis: biological and physical. Curr Opin Rheumatol 2014;26:704-11.

14. Amato AA, Barohn RJ. Evaluation and treatment of inflammatory myopathies. J Neurol Neurosurg Psychiatry 2009;80:1060-8.

15. Catoggio LJ, Managemant of inflammatory muscle disease. In: Hochberg MC, Silman AJ, Slomen JS, Weinblatt ME, Weisman MH, editors. Rheumatology. Philadelphia: Mosby (Elsevier); 2007. p. 1461-8.

16. Bunch TW. Prednisone and azathioprine for polymyositis: long-term followup. Arthritis Rheum 1981;24:45-8.

17. Dalakas MC, Illa I, Dambrosia JM, Soueidan SA, Stein $\mathrm{DP}$, Otero $\mathrm{C}$, et al. A controlled trial of high-dose intravenous immune globulin infusions as treatment for dermatomyositis. N Engl J Med 1993;329:1993-2000.

18. Majithia V, Harisdangkul V. Mycophenolate mofetil (CellCept): an alternative therapy for autoimmune inflammatory myopathy. Rheumatology (Oxford) 2005;44:386-9. 
19. Efthimiou P, Schwartzman S, Kagen LJ. Possible role for tumour necrosis factor inhibitors in the treatment of resistant dermatomyositis and polymyositis: a retrospective study of eight patients. Ann Rheum Dis 2006;65:1233-6.
20. Miller FW, Leitman SF, Cronin ME, Hicks JE, Leff $\mathrm{RL}$, Wesley $\mathrm{R}$, et al. Controlled trial of plasma exchange and leukapheresis in polymyositis and dermatomyositis. N Engl J Med 1992;326:1380-4. 